\title{
Loss of Expression of Cyclin D2 by Aberrant DNA Methylation: a Potential Biomarker in Vietnamese Breast Cancer Patients
}

\author{
Phuong Kim Truong ${ }^{1}$, Thuan Duc Lao ${ }^{1}$, Thao Phuong Thi Doan ${ }^{2}$, Thuy Ai Huyen Le ${ }^{1 *}$
}

\begin{abstract}
DNA methylation of tumor suppressor gene promoters is the most frequent phenomenon leading to inactivation of function, consequently driving malignant cell transformation. Cyclin D2 is implicated in tumor suppression. In our study, we carried out the MSP assay to evaluation the methylation status at CpG islands in the cyclin $D 2$ promoter in breast cancer cases from the Vietnamese population. The results showed that the frequency of methylation reached $62.1 \%$ (59 of 95 breast cancer tumors), but was low in non-cancer specimens at $10 \%$ ( 2 of 20 non-cancer specimens). Additionally, with an RR (relative risk) and OR (odd ratios) of 6.21 and 14.8, DNA hypermethylation of cyclin D2 increased the possibility of malignant transformation. Our results confirmed the cyclin D2 hypermethylation could be used as the potential biomarker which could be applied in prognosis and early diagnosis of Vietnamese breast cancer patients.
\end{abstract}

Keywords: Cyclin D2 - methylation - MSP - breast cancer - Vietnamese population

Asian Pac J Cancer Prev, 16 (6), 2209-2213

\section{Introduction}

Cyclin D2, a protein belonging to the highly conserved cyclin family, is characterized as the regulator of the cell cycle at the point of transition from $\mathrm{G} 1$ to $\mathrm{S}$ phase during the cell cycle (Evron et al., 2001; Yu et al., 2003). Its critical function is involved in forming a complex with the subunit of CDK4 and CDK6, subsequently, leading to the phosphorylation of RB (retinoblastoma). This allows the releasing of the transcription factor E2F from the RB/ E2F complex, in turn, activates the transcription of genes involved in DNA synthesis which are responsible for the progression through the G1 to S phase (Zhang, 1999; Evron et al., 2001).

Regarding to their critical roles in cell cycle regulation, the abnormal and untimely expression could lead to the disruption of the cell cycle progression. Cyclin D2 has been indicated that it is overexpressed in various cancers including lymphomas, breast cancer, lung and thyroid cancer... as the role of oncogene (Zhang, 1999). Of note, as the role of tumor suppressor gene (TSG), the loss of cyclin D2 expression is observed in several types of cancer including prostate cancer (Padar et al., 2003), small cell/ non-small cell lung cancer, breast cancer (Virmani et al., 2003, Salskov, 2011), gastric cancer (Yu et al., 2003), colorectal cancer (Tiagno et al., 2013) etc. A common event in the silencing of TSG is associated to the DNA methylation of at the promoter regions. DNA methylation, is the covalent addition of the methyl group to Cytosine residues of the $\mathrm{CpG}$ dinucleotide in DNA, which plays an important role in driving tumorgenesis, including breast carcinoma (Pongtheerat et al., 2011; Ramezani et al., 2012; Phuong et al., 2014). Especially, the inactive of cyclin D2 gene, is contributed to hypermethylation of the target gene promoter region, has been reported in breast cancer as the early event in malignant breast cancer transformation (Evron et al., 2001; Virmani et al., 2003). The methylation frequency of cyclin D2 were $32 \%, 52 \%$ and $23 \%$ in invasive lobular carcinoma, invasive ductal carcinoma, lobular carcinoma in situ, respectively (Fackler et al., 2003), 11\% in general breast cancer ( $\mathrm{Li}$ et al., 2006). According to those results, even though methylation of cyclin D2 in breast cancer is not so high, but the methylation of cyclin D2 is the specific characteristic of breast cancer.

Recently, de-methylating agents have been approved for the aim of reversing gene silencing mediated by DNA methylation, in which, the hypermethethylation at the $\mathrm{CpG}$ island at promoter region of cyclin D2 was chosen as a potential candidate for testing. For examples, anastrozole effected on the promoter's de-methylation of a panel of genes including cyclin D2 (Prowell et al., 2008); Sulforaphane de-methylates promoter of cyclin D2 in prostate cancer (Anna Hsu et al., 2011) or 5-aza-2'deoxycytidine (decitabine, 5-Aza-CdR) was approved that it was an inhibitor of DNA methyltransferase 1 leading 
to increase the expression of p16INK4a and p19INK4d, the products of CDKN2A and CDKN2D (Zhu et al., 2001 ); etc. Thereby, to elucidate the methylation status of involved gene in tumorgenesis is a promising target for testing of the epigenetic therapy as the demethylatingepidrugs.

Up to now, no any study about the hypermethylation of Cyclin D2 carried out on Vietnamese population. Therefore, for the aim to investigate whether Cyclin D2 silencing in breast cancer by mechanism of DNA hypermethylation. in present study we examined the methylation status of cyclin D2 promoter in Vietnamese breast cancer patients of all stages from premalignant to advanced metastatic breast tumor and comparison with healthy specimens by the MSP (methylation specific PCR) method.

\section{Materials and Methods}

\section{Clinical samples collection}

The total of 115 samples composed of 95 breast cancer specimens and 20 healthy specimens used in this study were collected and admitted from the Ho Chi Minh city Medical Hospital in Vietnam from 2010 to 2011. All breast cancer specimens were enrolled in evaluating the predictive factors including immunohistochemistry with two antibodies including Her2/neu and p53. Moreover, the histologic grade and tumor grade of tumor specimens were also determined. Besides, the healthy samples were noncancer tissue which were collected from healthy patient. Those patients had undergone to get the well information about the clinical and molecular characteristics of the tumors. Firstly, the age of patients were ranged from 25 to 88 years old, the median was 48 years old. For the two immunochemistry antibodies, Her2 positive and p53 positive were detected in $59(62.1 \%)$ and $17(17.0 \%)$, respectively. The sum of sample with both Her2 and p53 negative was counted for 33 of $95(28.7 \%)$ breast cancer specimens. Otherwise, the both Her2 and p53 positive was $82(71.3 \%)$. Moreover, the stages of tumors were large in stage I, II, III and IV which were accounted for $53(55.8 \%), 27(28.4 \%), 9(9.5 \%)$ and $6(6.3 \%)$ of 95 specimens. According to the tumor grade, grade 1,2 and 3 were $5(5.3 \%), 55(57.9 \%), 35(36.8 \%)$ of 95 samples, respectively. These tissues were embedded in the paraffin and stored at $-20^{\circ} \mathrm{C}$ until the further used.

\section{DNA extraction, DNA bisulfite modification and Methylation assays}

Genomic DNA was extracted from paraffin embedded tissues by proteinase $\mathrm{K}$ digestion (Life technologies, AM2546) and Phenol/chloroform extraction and ethanol precipitation as previous descried (Chomczynski and Sacchi, 1987). The quality and purity of DNA was determined by the proportion of A260/A280. Then, the DNA solution was stored at $-20^{\circ} \mathrm{C}$ until further bisulfite modification and MSP assay. For DNA bisulfite modification, DNA modification kit (Epitech Kit, Qiagen, 59110) was used in this study. Approximate $2 \mu \mathrm{g}$ genomic DNA of each sample was bisulfite modified and purified, then, the final precipitate was eluted in a volume of 20 $\mu l$ according to the manufacturer's instruction. Finally, bisulfite-modified DNA was amplified by methylate Specific PCR with the methylated and unmethylated primers were given in Table 1. Besides, the cyclin D2 sequence, $\mathrm{CpG}$ sites and transcriptional factor such as $<$ GATA-2, >C/EBP, Sp1,... were shown in Figure 1.

For the MSP assay, the amplifications were done in a total volume of $15 \mu \mathrm{l}$, containing $3 \mu \mathrm{l}$ bisulfite modified template DNA, 0.75 unit iTaq DNA polymerase (Biorad). MSP reaction was subjected to initial incubation at $95^{\circ} \mathrm{C}$ for $5 \mathrm{~min}$, followed by 40 cycles at $95^{\circ} \mathrm{C}$ for $30 \mathrm{~s}, \mathrm{X}^{\circ} \mathrm{C}$ for $30 \mathrm{~s}\left(\mathrm{X}=56^{\circ} \mathrm{C}\right.$ for methylated primer, $\mathrm{X}=54^{\circ} \mathrm{C}$ for unmethylated primer), $72^{\circ} \mathrm{C}$ for $30 \mathrm{~s}$ and $72^{\circ} \mathrm{C}$ for $6 \mathrm{~min}$ for final incubation. Each PCR product was directly loaded onto a $2.0 \%$ agarose gel, stained with ethidium bromide, and directly visualized under UV illumination.

\section{Statistical analysis}

The frequency of methylation of cyclin D2 gene were calculated. Differences in the presence of methylation/ unmethylation were determined by a two sided Fisher test and Chi squared tests for variables. The relative risk (RR), associations between the methylated status of $\mathrm{cyclin}$ D2 and breast cancer, as well as any correlation between methylation status and other clinical parameters were examined using the Chi-square test. Moreover, the Odd ratio (OR) and $95 \%$ confidence intervals (CIs) were also

Table 1. Methylation and Inmethylation Cyclin D2 Primer

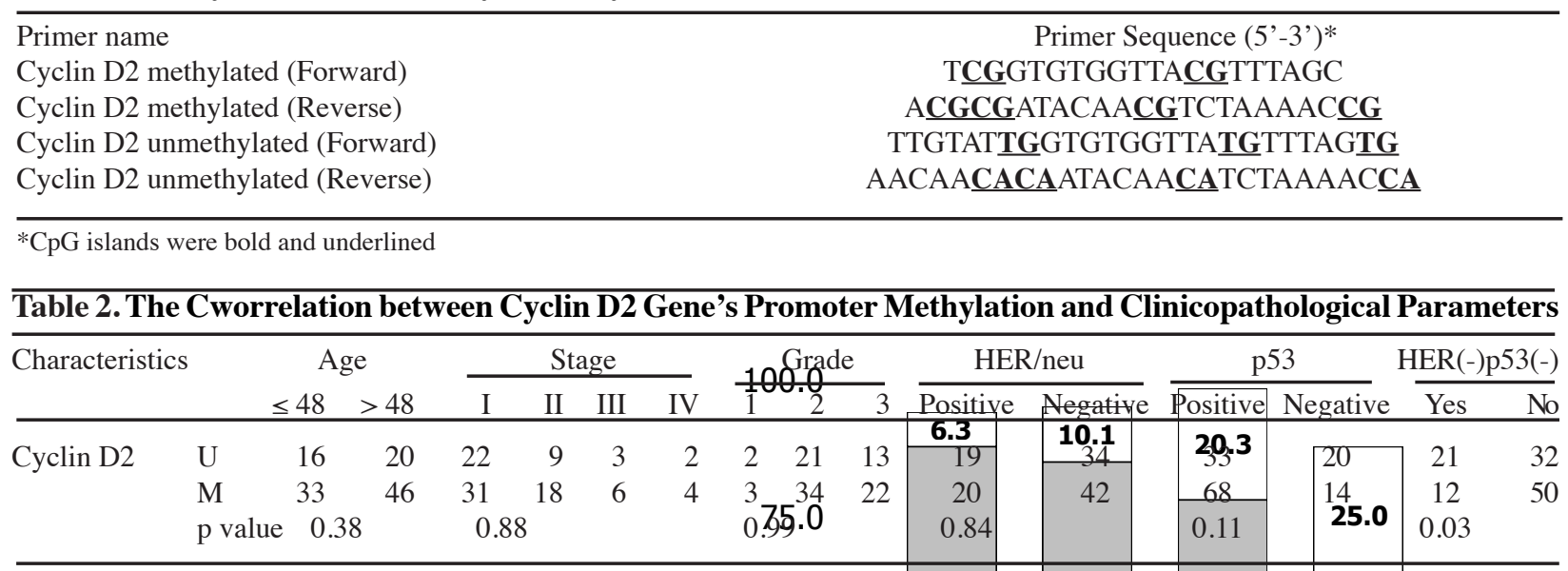




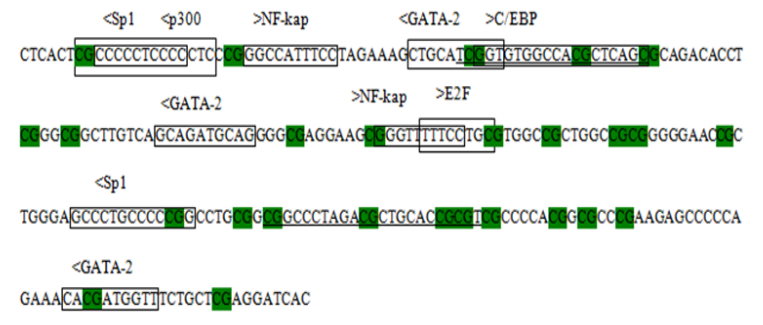

Figure 1. The Schematic Representation of Cyclin D2 Promoter with the MSP Primer and Transcriptional Factor Binding Sites. CpG sites are green and underlined nucleotides are chosen for MSP primer designed. Underlined nucleotides are chosen for primer designed
(A)

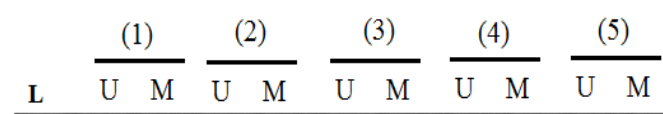

(B)

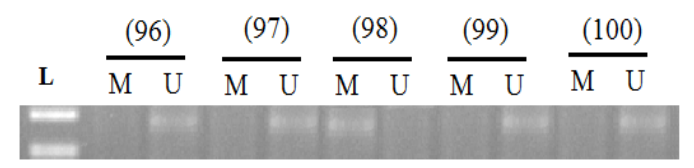

Figure 2. The Amplification of Cyclin D2 Promoter on Some Clinical Samples by MSP Method. (A) Breast cancer specimens; (B) Non-cancer specimens

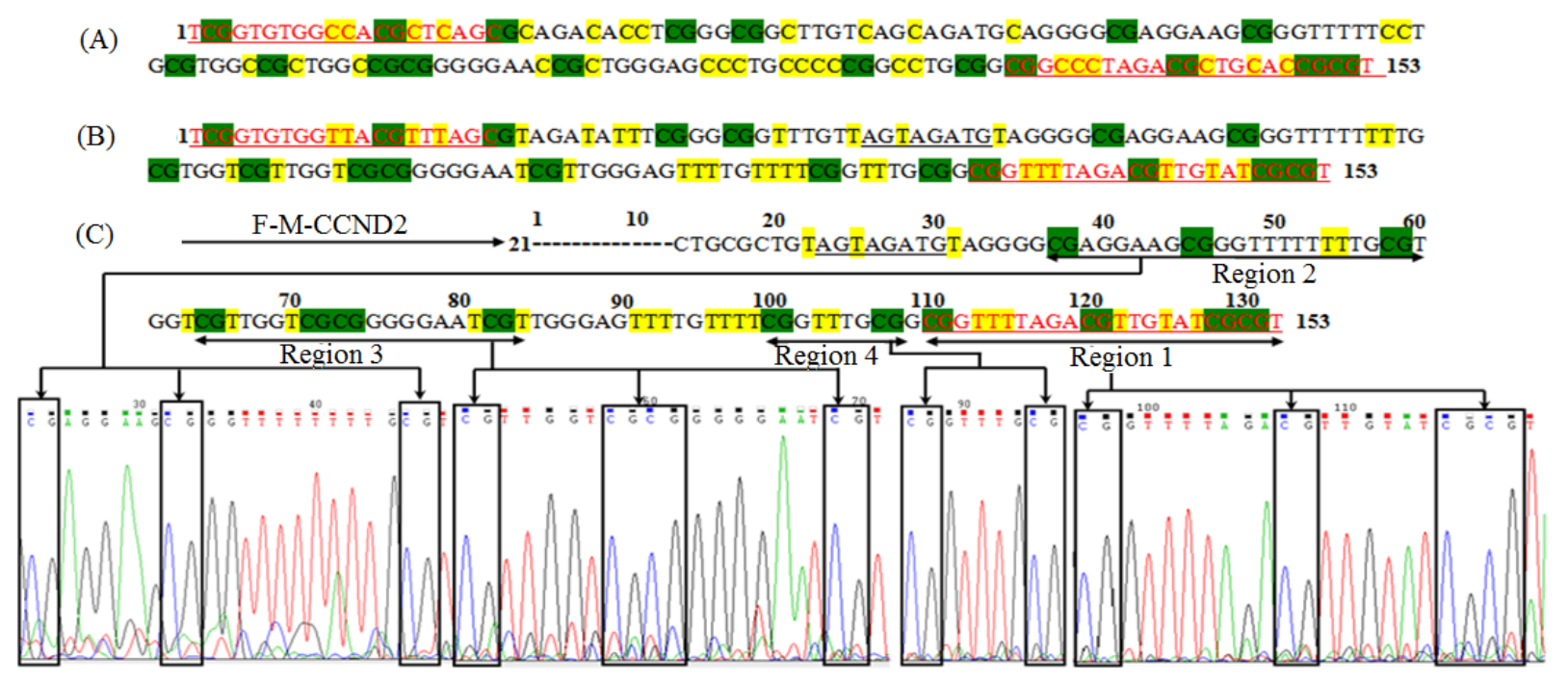

Figure 3. Sequencing Profile of Methylated Promoter of Cyclin D2. The Cytosine did not depend on the CpG sites were in yellow. The CpG sites were in green. (A) DNA sequence was without bisulfite modified; (B) DNA sequence was bisulfite modified; (C) The MSP Cyclin D2 was sequenced. Region 1: R-M-CCND2 binding region containing 4 CpG sites; Region 2: Three CpG sites were in examined region; Region 3: Four CpG sites were in examined region; Region 4: Two CpG sites were in examined region.

calculated. Statistical analyses were performed by using Medcalc ${ }^{\circledR}$ Version 12.7.0.0. Statistical significance was assumed at two-side $\mathrm{p}$ value of $\mathrm{p}<0.05$.

\section{Results}

Cyclin D2 promoter hypermethylation in breast cancer

The frequency of Cyclin D2 promoter methylation was evaluated by MSP assay on total of 115 samples including breast cancer specimens and healthy samples. The frequency of methylation status was $62.1 \%$ (59 cases of 95 cancer specimens $)(\mathrm{p}<0.0001)$. Otherwise, in healthy samples, only 2 of 20 cases were identified in methylation status, counting for $10 \%$. The MSP product, the length of $153 \mathrm{bp}$, was directly loaded onto a $2.0 \%$ agarose gel under the UV illumination (Figure 2), then, sequencing was carried out to confirm the accuracy of the target gene amplification (Figure 3).

The correlation between hypermethylation promoter Cyclin D2 and several clinicopathological parameters

The table 2 showed that there were no any significant differences between the hypermethylation promoter Cyclin D2 and clinicopathological characteristics including patients' age, stage and tumor grade, p53 and Her/neu expression $(p>0.05)$. Besides, the significant correlation was found out between the hypermethylation status of Cyclin D2 promoter and Her(-)p53(-) characteristic $(\mathrm{p}=0.03)$ (Table 2). Moreover, the relative risk and odd ratio were calculated, which were $6.2105(\mathrm{p}=0.007)$ and $14.75(\mathrm{p}<0.001)$, respectively.

\section{Discussion}

In mammalian cells, the transition from $\mathrm{G} 1$ phase to $\mathrm{S}$ phase is regulated by cyclin D in combination with Cdk (cyclin dependent kinase). Therefore, they play distinct roles in cell progression through the cell life. Moreover, as mentioned above, it has demonstrated that the frequent loss of cyclin D2 expression is associated with the driving to carcinomas. The loss of Cyclin D2 is strongly correlated to the hypermethylation of its promoter (Evron et al,2001; Fackler et al., 2003; Virmani et al, 2003; Yu et al, 2003; Padar et al, 2003; Li et al, 2006) and this phenomenon is the early event in tumorgenesis including breast cancer (Evron et al, 2001; Virmani et al, 2003). So that, it is interesting in determining whether methylation of cyclin D2 gene promoter during an early stage of malignant transformation in breast cancer and can serve as the early marker for breast cancer detection. 
In this study, we have successfully carried out the MSP method to evaluate the methylated status of cyclin D2 promoter. At first, for picking MSP primer, Methprimer and TFSearch program were applied in appraising CpG islands and transcriptional factor located in cyclin D2 promoter sequence. These transcriptional factors binding sites including $<$ GATA-2, $>$ C/EBP, Sp1, etc were situated in promoter are evidently associated with gene expression in human. (Uhm et al, 2012). Therefore, these sites are clearly ideal regions for picking MSP primers since the aberrantly hypermethylated $\mathrm{CpG}$ ' $\mathrm{C}$ 's in or near these transcriptional binding sites could probably directly lead to the inactivation of transcription. MSP primers were designed to have at least three $\mathrm{CpG}$ sites, one $\mathrm{CpG}$ site preferable at most 3'-end. Each methylated and unmethylated primer set were chosen to cover seven $\mathrm{CpG}$ and cover at least two transcriptional factor binding sites. Those primers were used to evaluate the methylated and unmethylated status of cyclin D2 promoter, the results of MSP analysis were confirmed by sequencing. By sequencing, we observed total of nine $\mathrm{CpG}$ islands which were totally methylated in region 2, 3 and 4. Additionally, in the region 1 , we detected four $\mathrm{CpG}$ sites methylated where the reverse methylated primer binding. Besides, it could be observed that the signal of peaks in MSP product sequencing were unique, clear and specific. Therefore, according to these results, it could be concluded that the bisulfite modification was successfully carried out.

The methylation status in tumor specimens were frequent, in our report, accounting for $62.1 \%$ (59 cases of 95 cancer specimens). Comparison with other reports, the mean of frequency of hypermethylated cyclin D2 was higher than the research of Evron et al (2001) as $46 \%$, two times higher than Virmani et al (2003) as 30\% in breast tumor tissue, and higher than Li et al (2006) as $11 \%$ in breast tumors. Interestingly, the similar to those studies could be observed on evaluating the methylation or unmethylation in the non-cancer specimens. Hence, it could be induced that the hypermethylation at $\mathrm{CpG}$ site belonged to the cyclin D2 promoter is the specific outcome characteristic of Vietnamese breast cancer. Regarding to the two most common genetic abnormalities associated with breast cancer including Her2/neu and p53, we found that there were poor connection between them $(p>0.05)$. However, taken these two biomarkers together, the strong negative correlation between Her2/neu(-)p53(-) and the hypermethylation status $(\mathrm{p}=0.03)$. If noted, percentage of Her2/neu(-)p53(-) was counting for over $70 \%$ (82 of 115 samples) in which, the cases with positive of methylation was 50 of 82 samples (accounting for more than $60 \%$ ). Therefore, we tentatively suggest that in the case of the patients with the negative of both Her $2 /$ neu and p53, the positive hypermethylation of cyclin D2 will be the potential screening factor for high risk breast cancer prediction. It also could be referred that the further transformation to malignant cell in following several years had to be considered and there will be the critical key for us to get more data in future to evaluate the status of hypermethylation of cyclin D2 in early stage of breast cancer transformation.

Besides, the RR and OR were calculated to confirm the ability of hypermethylation cyclin D2 promoter could be serve as the potential for prognosis and early diagnosis for Vietnamese breast cancer. The RR and OR were $6.21(\mathrm{p}=0.007,95 \% \mathrm{CI})$ and $14.75(\mathrm{p}<0.001,95 \% \mathrm{CI})$, respectively. It meant that the candidate gene promoter methylated was strongly correlated with breast cancer risk via the RR and OR with the significant statistic. It was also meant that the odds for a positive hypermethylation of cyclin D2 promoter in breast cancer was 14.75 times higher than in the case of cancer without methylation. More, the hypermethylation of candidate gene was 6.21 times higher than unmethylation in breast tumor. Based on these data, it could be strongly to note that the $\mathrm{CpG}$ aberrant methylation of Cyclin D2, as the role of tumor suppressor gene, leading to the gene silencing, was the critical key of driving the breast tumorgenesis and could be detected in the early stage of malignant transformation. For those reasons, cyclin D2 is the potential candidate gene for studying the epigenetic based cancer therapy in Vietnam.

In conclusion, 95 tumors from Vietnamese breast cancer patient and 20 non-cancer specimens were used in evaluation of cyclin D2 promoter methylation analysis. It was highlight noted that the frequency reached to $62.1 \%$ in tumor specimens and $10 \%$ in non-cancer specimens. This aberrant methylation leading to the gene silencing was the specific outcome of breast cancer patients, especially, significant associated with the Her2/neu(-)p53(-) stage, except the patient age, tumor grade and stage. Additionally, the OR, RR were 14.75 and 6.21, respectively. These data could be included that the tumor suppressor gene, cyclin $D 2$, silencing by hypermethylation promoter could be used as a potential biomarker for Vietnamese breast cancer prognosis and early diagnosis. Theyalso suggested that further studies are reasonable to follow and carry out more researches about the mechanism of methylated cyclin D2 gene promoter by the demethylation drug in cancer therapy..

\section{Acknowledgements}

This study was funded by The Department of Science and Technology, Ho Chi Minh City, Vietnam.

\section{References}

Anna Hsu, Carmen P Wong, Zhen Yu, et al (2011) Promoter demethylation of cyclin D2 by sulforaphane in prostate cancer cells. Clin Epigenetics, 3, 3 .

Chomczynski P, Sacchi N (1987). Single-step method of RNA isolation by acid guanidinium thiocyanate-phenolchloroform extraction. Anal Biochem, 162, 156-9.

Evron E, Umbricht CB, Korz D, et al (2001). Loss of cyclin D2 expression in the majority of breast cancers is associated with promoter hypermethylation. Cancer Res, 61, 2782-7.

Fackler MJ, McVeigh M, Evron E, et al (2003). DNA methylation of RASSF1A, HIN-1, RAR-beta, Cyclin D2 and Twist in in situ and invasive lobular breast carcinoma. Int J Cancer, 107, 970-5.

Li S, Rong M, Iacopetta B (2006). DNA hypermethylation in breast cancer and its association with clinicopathological features. Cancer Lett, 237, 272-80. 
Padar A, Sathyanarayana UG, Suzuki M, et al (2003). Inactivation of cyclin $\mathrm{D} 2$ gene in prostate cancers by aberrant promoter methylation. Clin Cancer Res, 9, 4730-4.

Pongtheerat T, Pakdeethai S, Purisa W, Chariyalertsak S, Petmitr $S$ (2011). Promoter methylation and genetic polymorphism of glutathione S-transferase P1 gene (GSTP1) in Thai breastcancer patients. Asian Pac J Cancer Prev, 12, 2731-4.

Prowell TM, Fackler M, Argani P, et al (2008). A prospective study of adjuvant anastrozole and gene promoter methylation in the contralateral breast of high-risk women. J Clin Oncol, 26, 1514 .

Ramezani F, Salami S, Omrani MD, Maleki D (2012). CpG island methylation profile of estrogen receptor alpha in Iranian females with triple negative or non-triple negative breast cancer: new marker of poor prognosis. Asian Pac J Cancer Prev, 13, 451-7.

Truong PK, Lao TD, Doan TPT, Le TAH (2014). BRCA1 promoter hypermethylation signature for early detection of breast cancer in the Vietnamese population. Asian Pac J Cancer Prev, 15, 9601-10.

Uhm TG, Lee SK, Kim BS, et al (2012). CpG methylation at GATA elements in the regulatory region of CCR3 positively correlates with CCR3 transcription. Exp Mol Med, 44, 268-80

Virmani A, Rathi A, Heda S, et al (2003). Aberrant methylation of the cyclin D2 promoter in primary small cell, non-small cell lung and breast cancers. Int J Cancer, 107, 341-5.

Yu J, Leung WK, Ebert MP, et al (2003). Absence of cyclin D2 expression is associated with promoter hypermethylation in gastric cancer. Br J Cancer, 88, 1560-5.

Zhang P (1999). The cell cycle and development: redundant roles of cell cycle regulators. Curr Opin Cell Biol, 11, 655-62

Zhu WG, Dai Z, Ding H, et al (2001). Increased expression of unmethylated CDKN2D by 5-aza-2'-deoxycytidine in human lung cancer cells. Oncogene, 20, 7787-96. 